\title{
QUARK-HADRON DUALITY: RESONANCES AND THE ONSET OF SCALING
}

\author{
W. MELNITCHOUK \\ Special Research Centre for the Subatomic Structure of Matter, \\ Adelaide University, 5005, Australia, and \\ Jefferson Lab, 12000 Jefferson Avenue, Newport News, VA 23606, USA
}

\begin{abstract}
We examine the origin of Bloom-Gilman duality and the relationship between resonances and scaling in deep-inelastic scattering. A simple quantum mechanical model is used to illustrate the essential features of Bloom-Gilman duality at low momentum transfer. As an application of local duality, we discuss model-independent relations between structure functions at $x \sim 1$ and elastic electromagnetic form factors.
\end{abstract}

\section{Introduction}

Quark-hadron duality addresses some of the most fundamental issues in strong interaction physics, providing a focus on the nature of the transition from the perturbative to nonperturbative regions of QCD. In its broadest form, it postulates that calculations of physical quantities performed in either a partonic (quark-gluon) or hadronic basis should yield identical results. Although duality is in practice almost never realized exactly, there are rare cases where the average of hadronic observables can be described to good accuracy within a low order perturbative QCD treatment.

The correspondence between hadronic and partonic descriptions has been studied in a number of physifal processes, such as $e^{+} e^{-}$annihilation into hadrons 1 , heavy meson decayst, and inclusive electron-hadron scattering. It also forms the foundation on which the theoretical framework of QCD sum rules is based. 3 In this review, we shall discuss some recent progress made in unraveling the origin of duality in inclusive electron scattering, first observed some 30 years ago by Bloom and Gilman. 6 Before discussing Bloom-Gilman duality in more detail, however, it will be useful to briefly review the concept of duality as it first appeared in the context of hadronic reactions.

\section{Duality in Hadron-Hadron Scattering}

Historically, duality in strong interaction physics was originally formulated for hadron-hadron scattering, where it represented the relationship between the $s$ and $t$ channel behaviors of scattering amplitudes. At low energies, the scattering amplitude, $\mathcal{A}(s, t)$, could be well described by a sum over a few 
$s$-channel resonances, $\mathcal{A}(s, t)=\sum_{\text {res }} \mathcal{A}_{\text {res }}(s, t)$. At large $s$, in the region of densely packed and overlapping resonances, a $t$-channel partial wave series was more useful, and the high energy behavior of $\mathcal{A}(s, t)$ could be described in terms of $t$-channel Regge poles and cuts, leading to the well-known linear Regge trajectories, $\mathcal{A}_{\mathbb{R}}(s, t) \sim s^{\alpha(t)}$, where $\alpha(t)=\alpha(0)+\alpha^{\prime} t$. The puzzle confronting hadron physicists of the 1960s was how to merge these descriptions, especially at intermediate $s$.

In analyzing $\pi N$ scattering amplitudes in the framework of finite energy sum rules, Dolen, Horn \& Schmid observedE an equivalence or duality between $s$-channel resonances and $t$-channel Regge poles, $\mathbb{R}$ :

$$
\sum_{\text {res }} \mathcal{A}_{\text {res }}(s, t) \approx \sum_{\mathbb{R}} \mathcal{A}_{\mathbb{R}}(s, t) .
$$

Furthermore, as $s \rightarrow \infty$, a "local" version of the duality relation (11) was found to hold, $\mathcal{A}_{\text {res }}(s, t) \approx \mathcal{A}_{\mathbb{R}}(s, t)$.

A model which unified the low and high $s$ behaviors was subsequently presented by Veneziand, in which the scattering amplitude was found to behave as

$$
\mathcal{A}(s, t) \sim \frac{\Gamma(-\alpha(s)) \Gamma(-\alpha(t))}{\Gamma(-\alpha(s)-\alpha(t))},
$$

with poles at integer values of $\alpha(s)$ and $\alpha(t)$. At high energy the model therefore reproduced the correct Regge behavior, $\mathcal{A}(s \rightarrow \infty, t) \sim$ $\Gamma(-\alpha(t))\left(\alpha^{\prime} s\right)^{\alpha(t)}$.

A generalization of the $s$ and $t$ channel duality, suggested by Hararit, included both resonant and nonresonant background contributions to cross sections. In this "two-component duality", resonances were said to be dual to nondiffractive Regge pole exchanges, while the nonresonant background was dual to Pomeron exchange:

$$
\mathcal{A}=\sum_{\text {res }} \mathcal{A}_{\text {res }}+\mathcal{A}_{\text {bckgnd }}=\sum_{\mathbb{R}} \mathcal{A}_{\mathbb{R}}+\mathcal{A}_{\mathbb{P}} .
$$

For duality in inclusive electron scattering, observed shortly thereafter at SLAC, this naively translates into a duality between resonances and valence quarks (for which the small $x \sim 1 / s$ behavior is given by poles on the $J^{P C}=$ $1^{--}$Regge trajectory), with the background dual to sea quarks (whose small $x$ behavior is governed by Pomeron exchange). 


\section{Bloom-Gilman Duality}

In studying inelastic electron scattering in the resonance region and the onset of scaling, Bloom and Gilman found ${ }^{4}$ that the inclusive $F_{2}$ structure function at low $W$ generally follows a global scaling curve which describes high $W$ data, to which the resonance structure function averages. More recently, this behavior was confirmed in high precision measurements of the $F_{2}$ structure function in the resonance region at Jefferson Labl. The similarity between the averaged resonance and scaling structure functions implies that the lowest moment of $F_{2}$ is approximately independent of $Q^{2}$. Furthermore, the data clearly demonstrate that duality works remarkably well for each of the lowlying resonances to rather low values of $Q^{2}\left(\sim 0.5 \mathrm{GeV}^{2}\right)$, as Fig. 1 illustrates.

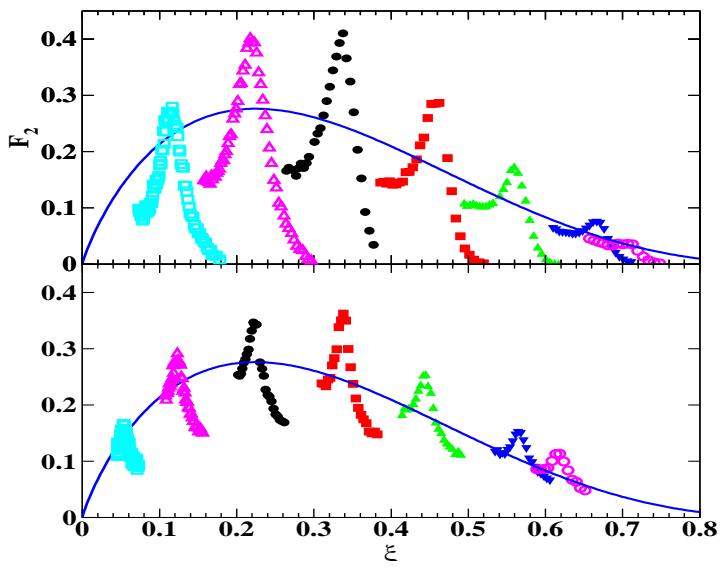

Figure 1. Proton $F_{2}$ structure function versus the Nachtmann scaling variable, $\xi$, in the first (upper panel) and second (lower panel) resonance regions, for ${ }^{\text {various }} Q^{2}$ between $0.07 \mathrm{GeV}^{2}$ (smallest $\xi$ ) and $3 \mathrm{GeV}^{2}$ (largest $\xi$ ), from Armstrong et al. 9 The solid line is the global scaling curve determined from all nucleon resonance data.

Before the advent of QCD, Bloom-Gilman duality was interpreted in the context of finite-energy sum rules 5 , in analogy with the $s$ and $t$ channel duality observed in hadron-hadron scattering. In QCD, Bloom-Gilman duality can be reformulated 1 in the language of the operator product expansion, in which moments of structure functions are organized in powers of $1 / Q^{2}$. The leading terms are associated with free quark scattering, and are responsible for scaling, while the $1 / Q^{2}$ terms involve interactions between quarks and gluons. The weak $Q^{2}$ dependence of the low moments of the structure function is then 
interpreted as indicating that the non-leading, $1 / Q^{2}$-suppressed, interaction terms do not play a major role even at low $Q^{2}$.

An important consequence of duality is that the strict distinction between the resonance and deep-inelastic regions becomes entirely artificial. To illustrate this, consider that at $Q^{2}=1 \mathrm{GeV}^{2}$ around $2 / 3$ of the total deep-inelastic cross section comes from the resonance region, $W<2 \mathrm{GeV}$. However, the resonances and the deep-inelastic continuum conspire to produce only about a $10 \%$ correction 11 to the lowest moment of the scaling $F_{2}$ structure function at the same $Q^{2}$. Even though each resonance is built up from a multitude of twists, when combined the resonances interfere in such a way that they resemble the leading twist component.

This can be even more dramatically illustrated by considering QCD in the large $N_{c}$ limit, where the hadron (or more specifically, meson) spectrum consists of infinitely narrow, noninteracting resonances. Since the quark level calculation still yields a smooth scaling curve, one sees that an averaging over hadrons must be invoked even in the scaling limit 12 . In the next section we demonstrate this duality explicitly in a simple model.

\section{Understanding the Origin of Duality}

The essential features of the dynamics behind Bloom-Gilman duality can be exposed with the help of a simple model in which the hadronic spectrum is dominated by infinitely narrom resonances made of valence quarks, as discussed recently by Isgur et al.2. To strip away irrelevant details which may unnecessarily complicate the illustration, one considers scattering from a relativistic scalar quark confined to an infinitely massive core by an oscillator-like potential. The virtue of such a model is that it affords exact solutions for the complete spectrum of excited states. Similar models have also been considered in Refs.13.14. Although clearly too simple to provide a realistic description of data, a model with these features nevertheless allows one to study the critical questions of when and why duality holds.

If all the excited states are infinitely narrow resonances, the structure function is given entirely by a sum of squares of transition form factors 22 ,

$$
\mathcal{S}\left(\nu, \vec{q}^{2}\right)=\sum_{N=0}^{N_{\max }} \frac{|\vec{q}|}{4 E_{0} E_{N}}\left|F_{0 N}(\vec{q})\right|^{2} \delta\left(E_{N}-E_{0}-\nu\right),
$$

where $E_{0}$ and $E_{N}$ are the energies of the ground state and $N$-th excited state, respectively, and $F_{0 N}$ is the transition form factor. The sum over $N$ is restricted to a maximum value, $N_{\max }$, allowed by the available energy transfer, 
$\nu$. Note that for a scalar probe, the structure function has dimensions of $(\text { mass })^{-2}$. The corresponding scaling variable,12 15

$$
u=\frac{1}{2 m}\left(\sqrt{\nu^{2}+Q^{2}}-\nu\right)\left(1+\sqrt{1+\frac{4 m^{2}}{Q^{2}}}\right),
$$

is defined with respect to the quark mass, $m$ (rather than the target mass, $M$, which is infinite), and takes into account nonzero mass effects at finite $Q^{2}$. In the limit $Q^{2} \rightarrow \infty$, the variable $u \rightarrow(m / M) x$.

Figure 2 shows the onset of scaling for the structure function $\mathcal{S}$ as a function of $u$ (the energy-dependent $\delta$-function has been smoothed out by a Breit-Wigner shape with a given width in order to display the $u$ dependence). With increasing $Q^{2}$ the resonances are seen to move out towards higher $u$, as observed in the physical spectrum in Fig. 1. Furthermore, the area under the curves remains approximately constant, indicating that global duality is reproduced by the model. Remarkably, the resonance spikes at lower $Q^{2}$ also tend to oscillate around the scaling curve, reminiscent of the local duality observed in the proton $F_{2}$ data.

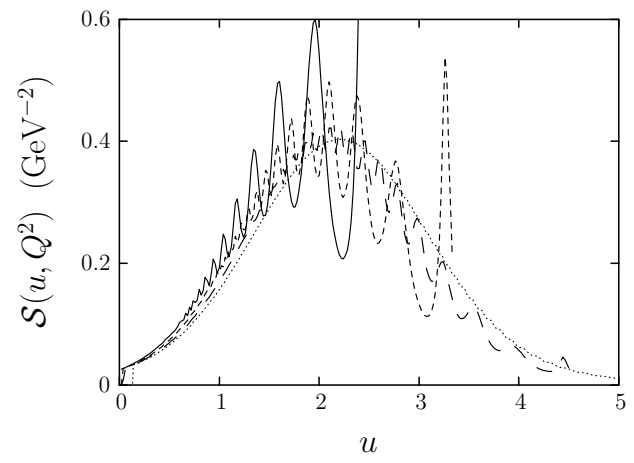

Figure 2. Scalar structure function versus the scaling variable $u$, for $22=0.5$ (solid), 1 (short-dashed), 2 (long-dashed) and $5 \mathrm{GeV}^{2}$ (dotted), from Isgur et al.12

Because the curves in Fig. 2 are at fixed $Q^{2}$, they necessarily span over a range of $\nu$ as $u$ varies. As $\nu$ increases, more thresholds for creating excited states are crossed (since $\vec{q}^{2}=Q^{2}+\nu^{2}$ ), and the density of states per $\vec{q}^{2}$ interval increases correspondingly. In fact, the correct density of states is crucial to compensate for the falling off with $\vec{q}^{2}$ of each individual form factor, $F_{0 N} \sim \vec{q}^{N} \exp \left(-\vec{q}^{2} / 4 \beta^{2}\right)$, where $\beta$ is related to the relativistic string constant 12 . Had the spacing between the excited states not been Regge-like 3 , $E_{N} \sim \sqrt{N}$, but linear as in a nonrelativistic solution, the density of states 
would not have been sufficient to produce duality. While it remains an interesting exercise to see whether potentials other than a harmonic oscillator give rise to similar behavior, this simple example illustrates that Bloom-Gilman duality appears as a natural consequence of even the most elementary quantum mechanical systems, with the only requirements being confinement and a correct treatment of kinematici 12 .

\section{Applications of Local Duality}

If the inclusive-exclusive connection via local duality is taken seriously, one can relate structure functions measured in the resonance region to electromagnetic transition form factors. 10 Isolating an individual resonance contribution to the inclusive structure function is problematic, however, since the separation of the resonance from the nonresonant background is modeldependent. For the extreme case of elastic scattering, on the other hand, there is no background below the pion production threshold, so the extraction of the elastic form factors from the inclusive structure function data (and vice versa) avoids this ambiguity.

The elastic magnetic form factor of the proton has in fact been extracted 1 from the recent Jefferson Lab data, and found to agree to within 30\% with the experimental form factor for $Q^{2}<5 \mathrm{GeV}^{2}$. Conversely, empirical electromagnetic form factors at large $Q^{2}$ can be used to predict the $x \rightarrow 1$ behavior of deep-inelastic structure functions $\theta$. Knowledge of structure functions at large $x$ is vital for several reasons - the $x \rightarrow 1$ behavior is very sensitive to mechanisms for spin-flavor SU(6) symmetry breaking, for instance, for which there are nonperturbative and perturbative QCD predictions. $16.1 \mathrm{t}$

Of particular interest is the polarization asymmetry, $A_{1}$, which at large $Q^{2}$ is given by the ratio of spin-dependent to spin-averaged structure functions, $A_{1} \approx g_{1} / F_{1}$. Assuming that the area under the elastic peak is the same as the area under the scaling function (atmuch larger $Q^{2}$ ) when integrated from the pion threshold to the elastic point 1 , the polarization asymmetry at threshold can be written as 18 :

$$
A_{1}\left(x_{\mathrm{th}}\right)=\left\{\frac{G_{M}\left(G_{M}-G_{E}\right)}{4 M^{2}(1+\tau)^{2}}+\frac{1}{1+\tau}\left(\frac{d\left(G_{E} G_{M}\right)}{d Q^{2}}+\tau \frac{d G_{M}^{2}}{d Q^{2}}\right)\right\} / \frac{d G_{M}^{2}}{d Q^{2}}
$$

where $x_{\mathrm{th}}=Q^{2} /\left(2 m_{\pi} M+m_{\pi}^{2}+Q^{2}\right)$, and $\tau=Q^{2} / 4 M^{2}$. In the limit $x_{\mathrm{th}} \rightarrow 1$, corresponding to $Q^{2} \rightarrow \infty$, both $F_{1}$ and $g_{1}$ are proportional to $d G_{M}^{2} / d Q^{2}$, so that $A_{1}^{p, n} \rightarrow 1$. Note that $\mathrm{SU}(6)$ symmetry predicts that for valence quarks $A_{1}=5 / 9$ for the proton and 0 for the neutron, while the perturbative QCD expectation based on helicity conservation is $A_{1}^{p, n} \rightarrow 1$ as $x \rightarrow 1$. 


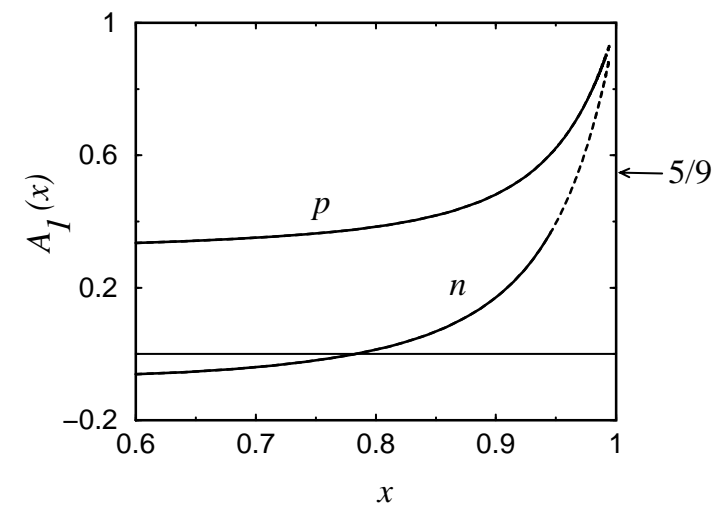

Figure 3. Polarization asymmetries $A_{1}$ for the proton and neutron at large $x$. The SU(6) predictions are $5 / 9$ for $p$ and 0 for $n$. The dashed extensions represent asymmetries calculated from extrapolations of form factors beyond the currently measured regions of $Q^{2}$.

Using parameterizations of global form factor data, we show in Fig. 3 the proton and neutron asymmetries $A_{1}$ as a function of $x=x_{\mathrm{th}}$. The solid curves represent the asymmetry calculated from actual form factor data, while the dashed extensions at larger $x$ illustrate the extrapolation of the form factors beyond the currently measured regions of $Q^{2}$. Unfortunately the current data on $A_{1}$ extend only out to an average $\langle x\rangle \sim 0.5$, and are inconclusive about the $x \rightarrow 1$ behavior. While the proton $A_{1}$ data do indicate a rise at $x \sim 0.5-0.6$, the neutron asymmetry is, within errors, consistent with zero over the measured range. It will be of great interest in future to observe whether, and at which $x$ and $Q^{2}$, the $A_{1}$ asymmetries start to approach unity.

Expressions similar to (6) can be derived also for other structure functions 18 . The ratios of the neutron to proton $F_{1}, F_{2}$ and $g_{1}$ structure functions are shown in Fig. 4 as a function of $x$, with $x$ again evaluated at $x_{\mathrm{th}}$. Asymptotically, each of the structure functions approaches $d G_{M}^{2} / d Q^{2}$, so that in the dipole approximation the $n / p$ ratios $\sim \mu_{n}^{2} / \mu_{p}^{2}$. Also indicated in Fig. 4 are some leading twist model predictions for the $F_{2}^{n} / F_{2}^{p}$, namely $2 / 3$ from $\mathrm{SU}(6)$ symmetry, $3 / 7$ from broken $\mathrm{SU}(6)$ with hejicity conservation, and $1 / 4$ from broken $\mathrm{SU}(6)$ with scalar diquark dominance 17 . Note, however, that the structure functions predicted from the duality relations contain both leading twist and higher twist contributions (for a discussion of the conditions under which the form factors can yield leading twist structure functions see Close \& Isgur 19 ). 


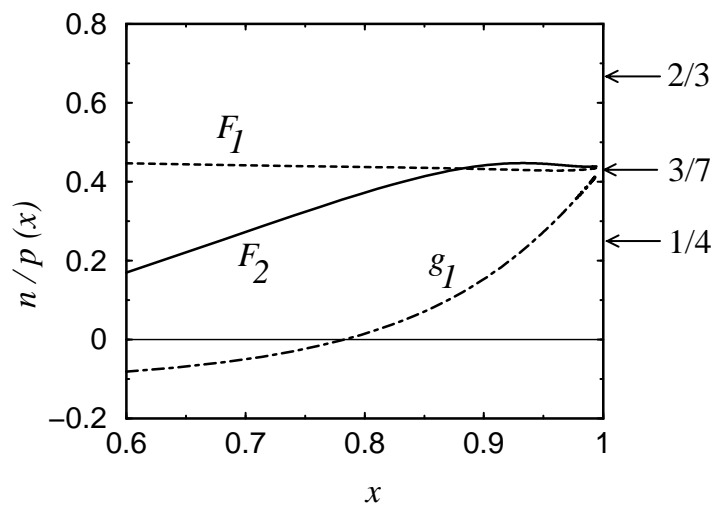

Figure 4. Neutron to proton ratio for the $F_{1}$ (dashed), $F_{2}$ (solid) and $g_{1}$ (dot-dashed) structure functions at large $x$. Several leading twist model predictions for $F_{2}$ in the $x \rightarrow 1$ limit are indicated by the arrows: $2 / 3$ from $\mathrm{SU}(6), 3 / 7$ from $\mathrm{SU}(6)$ breaking via helicity retention, and $1 / 4$ from $\mathrm{SU}(6)$ breaking through $d$ quark suppression.

The reliability of the duality predictions is of course only as good as the quality of the empirical data on the electromagnetic form factors and resonance structure functions. While the duality relations are expected to be progressively more accurate with increasing $Q^{2}$, the difficulty in measuring form factors at large $Q^{2}$ also increases. More data on form factors at larger $Q^{2}$ would allow more accurate predictions for the $x \rightarrow 1$ structure functions, and new experiments at Jefferson Lab and elsewhere will provide valuable constraints.

\section{Conclusion}

Quark-hadron duality offers the prospect of addressing the physics of the transition from the strong to weak coupling limits of $\mathrm{QCD}$, where neither perturbative QCD nor effective hadronic descriptions such as chiral perturbation theory are applicable. While considerable insight into quark-hadron duality has been gained from recent theoretical studies, it will be important in future to understand more quantitatively the features of the electron scattering data in the resonance region and the phenomenological $N^{*}$ spectrum in terms of realistic models of QCD.

On the experimental side, the spin and flavor dependence of duality can be most readily accessed through semi-inclusive scattering, which requires both high luminosity and a high duty factor. An energy upgraded Jefferson Lab 
would be an ideal facility to study meson production in the current fragmentation region at moderate $Q^{2}$, allowing the onset of scaling to be tracked in the pre-asymptotic regime. This would shed considerable light on the relationship between incoherent (single quark) and coherent (multi-quark) processes, and on the nature of the quark $\rightarrow$ hadron transition in QCD.

\section{Acknowledgements}

I am grateful to N. Isgur, S. Jeschonnek and J.W. Van Orden for their collaboration on the work presented in section 4 , and to experimentalists at Jefferson Lab for stimulating discussions on duality. This work was supported by the Australian Research Council and the U.S. Department of Energy contract DE-AC05-84ER40150, under which the Southeastern Universities Research Association (SURA) operates the Thomas Jefferson National Accelerator Facility (Jefferson Lab).

\section{References}

1. E.C. Poggio, H.R. Quinn and S. Weinberg, Phys. Rev. D 13, 1958 (1976).

2. M.B. Voloshin and M.A. Shifman, Sov. J. Nucl. Phys. 47, 511 (1988); N. Isgur and M.B. Wise, Phys. Lett. B 232, 113 (1989); N. Isgur, Phys. Rev. D 40, 101 (1989); Phys. Lett. B 448, 111 (1999); I.I. Bigi, M.A. Shifman, N.G. Uraltsev and A.I. Vainshtein, Phys. Rev. Lett. 71, 496 (1993).

3. M.A. Shifman, A.I. Vainshtein and V.I. Zakharov, Nucl. Phys. B147, 385 (1979); A.V. Radyshkin, in Strong Interactions at Low and Intermediate Energies, ed. J.L. Goity (World Scientific, 2000), hep-ph/0101227.

4. E.D. Bloom and F.J. Gilman, Phys. Rev. Lett. 16, 1140 (1970).

5. R. Dolen, D. Horn and C. Schmid, Phys. Rev. Lett. 19, 402 (1967).

6. G. Veneziano, Phys. Rept. 9, 199 (1974).

7. H. Harari, Phys. Rev. Lett. 20, 1395 (1969).

8. I. Niculescu et al., Phys. Rev. Lett. 85, 1182 (2000).

9. C.S. Armstrong et al., Phys. Rev. D 63, 094008 (2001).

10. A. de Rújula, H. Georgi and H.D. Politzer, Ann. Phys. 103, 315 (1975).

11. X. Ji and P. Unrau, Phys. Rev. D 52, 72 (1995).

12. N. Isgur, S. Jeschonnek, W. Melnitchouk and J.W. Van Orden, Phys. Rev. D, in print, hep-ph/0104022.

13. G. Domokos, S. Koveni-Domokos and E. Schonberg, Phys. Rev. D 3, 1184 (1971). 
14. E. Pace, G. Salme and F.M. Lev, Phys. Rev. C 57, 2655 (1998); S.A. Gurvitz and A.S. Rinat, Phys. Rev. C 47, 2901 (1993); E. Pace, G. Salme and A.S. Rinat, Phys. Lett. B 325, 289 (1994); M.W. Paris and V.R. Pandharipande, nucl-th/0105076.

15. R. Barbieri, J. Ellis, M.K. Gaillard and G.G. Ross, Phys. Lett. 64 B, 171 (1976).

16. W. Melnitchouk and A.W. Thomas, Phys. Lett. B 377, 11 (1996).

17. N. Isgur, Phys. Rev. D 59, 034013 (1999).

18. W. Melnitchouk, Phys. Rev. Lett. 86, 35 (2001).

19. F.E. Close and N. Isgur, hep-ph/0102067. 